\title{
Relationships of Epichloë typhina isolates from different host grasses
}

\author{
C.L. SCHARDL ${ }^{1}$, A. LEUCHTMANN ${ }^{2}$ and B.A. MCDONALD \\ ${ }^{1}$ University of Kentucky, Department of Plant Pathology, 201 PSB, 1405 Veterans Dr., Lexington, KY 40546-0312, U.S.A. \\ ${ }^{2}$ ETH Zurich, Institute of Integrative Biology, Plant Ecological Genetics, CHN G29 Universitaetstrasse 16, CH-8092 Zurich, \\ Switzerland \\ ${ }^{3}$ ETH Zurich, Institute of Integrative Biology, Plant Pathology, LFW B16, Universitaetstrasse 2, CH-8092 Zurich, Switzerland \\ schardl@uky.edu
}

\begin{abstract}
Epichloë typhina comprises interfertile strains associated with several grass species in tribes Poeae, Aveneae and Brachypodieae. Molecular phylogenetics indicate that E. typhina is paraphyletic to E. sylvatica and E. clarkii. Although interfertility barriers are evident between E. typhina and E. sylvatica, E. clarkii is interfertile with E. typhina. However, E. clarkii is associated specifically with Holcus lanatus (Aveneae). We addressed the possibility that E. typhina is a complex of genetically isolated populations distinguishable by host specificity. Populations of E. typhina were sampled from three Cantons on a transect from southwest to northeast Switzerland, and from 1-3 host species in each Canton. No fewer than 20 isolates were obtained from each host at each location. The intron-rich 5'-end of the beta-tubulin gene was sequenced from each isolate, and all of the sequences were aligned for phylogenetic and coalescence analyses. There was no indication of geographic isolation of the populations, but no haplotypes (sequence variants) were shared between isolates from different hosts. Therefore, E. typhina appears to be a complex of genetically isolated sympatric populations that are specific for different hosts.
\end{abstract}

Keywords: Brachypodium species, Clavicipitaceae, Dactylis glomerata, Epichloë, epichloë endophytes, grasses, host specificity, molecular phylogenetics, Poa species, Poaceae, population genetics

\section{Introduction}

The Epichloë and Neotyphodium species are, respectively, sexual and asexual fungi that maintain constitutive, systemic symbioses with many grasses. These endophytes range from the more virulent and contagious sexual species, to mutualistic but asexual species that are only transmitted vertically. These fungal species and their host grasses have been an interesting model for evolution of symbiotic systems. Results of such studies indicate a strong relationship between host specificity and phylogenetic species. Phylogenetic inference of Epichlö̈ species relationships has mainly used sequences of intron-rich segments of genes encoding beta-tubulin $(t u b B)$, translation elongation factor 1-alpha (tefA), and actin (actG) (Craven et al. 2001). Distinct phylogenetic species of Epichlö̈ are associated with each of the host genera Brachyelytrum (Epichloë brachyelytri), Glyceria (Epichloë glyceriae), Elymus (Epichloë elymi), and Bromus (Epichloë bromicola). The phylogenetic species Epichloë amarillans is associated with closely related host genera Agrostis and Sphenopholis, and possibly also Calamagrostis and Cinna species, all within the tribe Aveneae. Whereas E. amarillans has been found only in North America, Epichloë baconii has been identified in Agrostis and Calamagrostis species in Europe. However, the sequenced isolate from Calamagrostis villosa groups separately from a clade of isolates from Agrostis species. Despite this apparent contradiction with the phylogenetic species concept, E. baconii has been circumscribed to include isolates from both genera because they are interfertile. In contrast, the phylogenetic species, Epichloë festucae, includes isolates from Festuca and Lolium that are interfertile, as well as a stromaforming isolate from Koeleria sp. that was not interfertile with the others. Yet, all E. festucae isolates to date have been highly similar in sequences of $a c t G$, tefl, and $t u b B$ introns, and the rDNA-ITS regions. Interestingly, E. typhina sensu stricto, which has been defined mainly on interfertility relationships, is associated with several genera in three different grass tribes: Poeae, Aveneae, and Brachypodieae (Leuchtmann \& Schardl 1998). This is a genetically diverse species, which is paraphyletic to Epichloë sylvatica and Epichloë clarkii. Although E. clarkii is interfertile with E. typhina, a morphological difference in the ascospores and its restriction to the host species Holcus lanatus have justified its specific binomial (White 1993). Likewise E. sylvaticum is restricted to a single host species, Brachypodium sylvaticum (Leuchtmann \& Schardl 1998). Interestingly, E. typhina isolates from the congener, Brachypodium pinnatum are closely related to E. sylvatica. Surprisingly, whereas $E$. sylvatica isolates appear not to be interfertile with those from $B$. pinnatum, the latter are interfertile with more distantly related E. typhina isolates associated with Dactylis glomerata (Craven et al. 2001; Leuchtmann \& Schardl 1998). Isolates of Epichloë spp. from different host genera almost never have identical $t u b B$, tef $A$ and $a c t G$ sequences sequences. To date, the only exceptions have been E. festucae isolates. The possibility that E. typhina is a single, broad host range species might need reconsideration if this pattern holds in a more extensive sampling of E. typhina isolates from several common hosts. Therefore, we conducted surveys of isolates from four hosts: Brachypodium pinnatum, Dactylis glomerata, Poa nemoralis, and Poa trivialis, in a transect of Switzerland, to determine the extent of gene flow between populations defined on a host basis.

\section{Methods}

Plants of $B$. pinnatum, D. glomerata, $P$. nemoralis and $P$. trivialis exhibiting choke disease were identified in several locations in France and Switzerland, on a transect from North $46^{\circ} 20.6^{\prime}$ East $6^{\circ} 04.9^{\prime}$ (near Vesancy, France) to North $47^{\circ} 45.7^{\prime}$ East $8^{\circ} 36.9^{\prime}$ (near Merishausen, Switzerland (Table 1). Stromata were selected from plants at least $1 \mathrm{~m}$ apart. Stromata were lyophilised

Table 1 Numbers of E. typhina isolates sampled and sequenced from each of four regions of France and Switzerland.

\begin{tabular}{|c|c|c|c|c|}
\hline \multirow{2}{*}{ Host } & \multicolumn{4}{|c|}{-------------- Location ${ }^{a}$------------- } \\
\hline & Vesancy & VD & $\mathrm{ZH}$ & $\mathrm{SH}$ \\
\hline B. pinnatum & - & - & 38 & - \\
\hline D. glomerata & 33 & 28 & 29 & 27 \\
\hline P. nemoralis & - & 26 & - & - \\
\hline P. trivialis & 7 & 29 & 43 & - \\
\hline
\end{tabular}

a Locations are Vesancy in France, and Cantons Vaud (VD), Zürich (ZH) and Schaffhausen (SH) in Switzerland. 
Figure 1 Phylogenetic relationships of Epichloë typhina isolates from populations of B. pinnatum, D. glomerata, P.nemoralis and $P$. trivialis based on comparisons of tubB intron sequences. Also included is the sequence from Epichloë clarkii, a species that is associated with Holcus lanatus and is infertile with E. typhina.

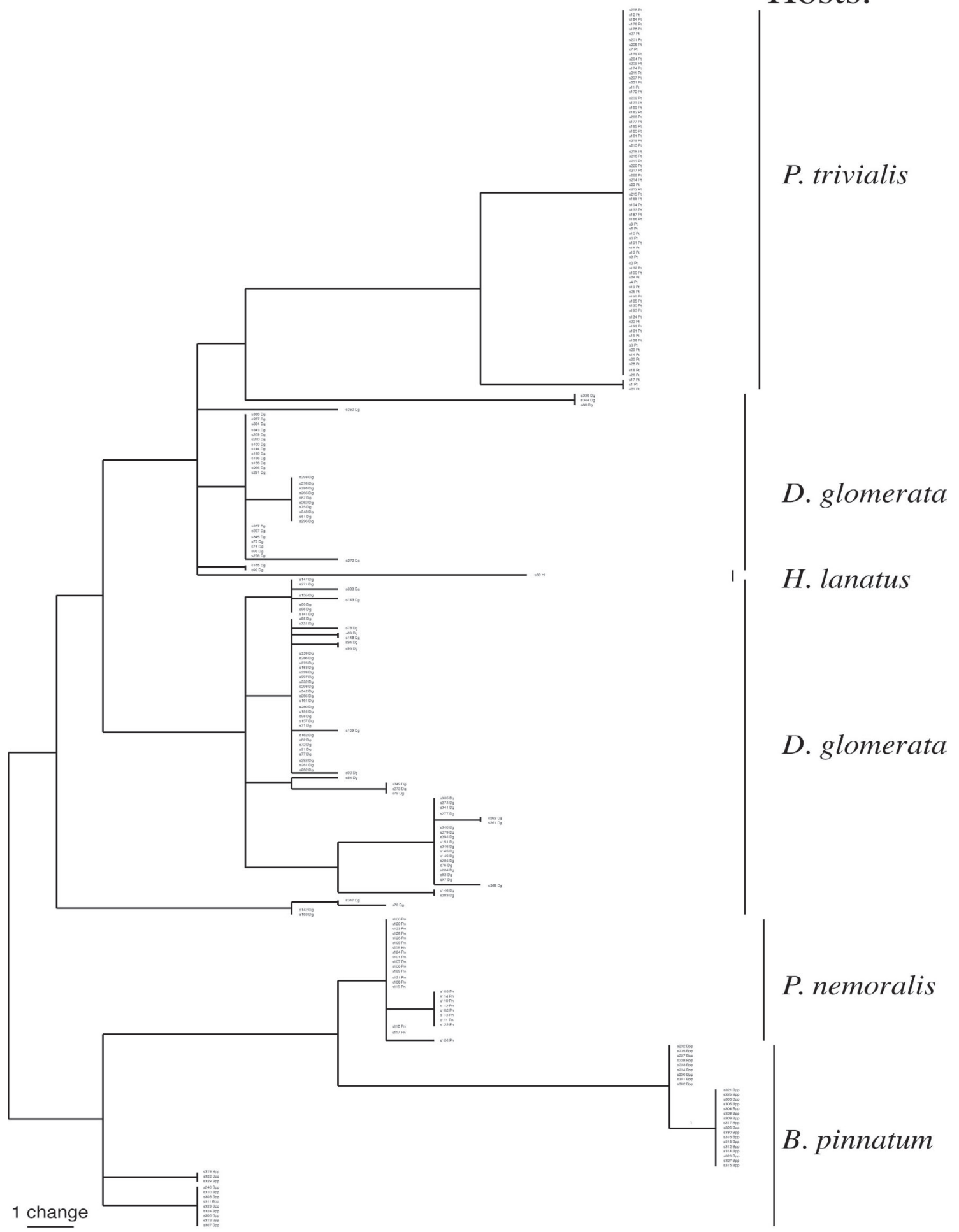


Figure 2 Phylogenetic relationships of $t u b B$ introns sequences from Epichloë species and selected Neotyphodium species. Each sequence is shown once, except those from $E$. festucae isolates from different host species. The LAE clade represents one of the genomes from four interspecific hybrids, for which the other alleles are not shown. Isolates in the E. typhina complex include those of E. clarkii and E. sylvatica, where indicated, or E. typhina as currently described.

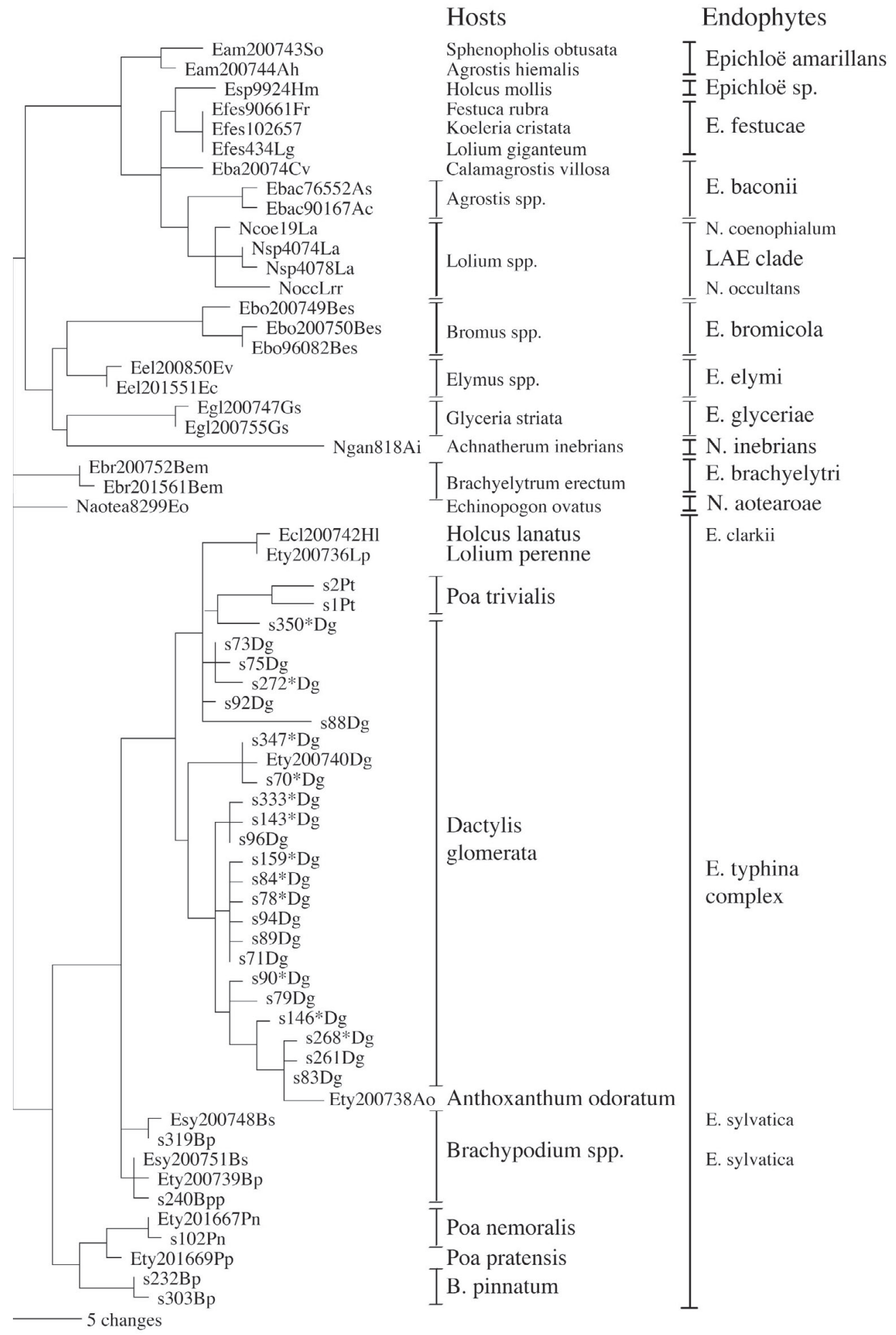


for storage and later DNA isolation. Stems from the stromabearing tillers were surface disinfested, then placed on nutrient agar medium to grow out endophyte cultures as previously described (Leuchtmann 1994; Leuchtmann \& Clay 1988). DNA was isolated from lyophilised stromata or fungal mycelium with the DNAeasy plant minikit or DNAeasy 96 plant kit from Qiagen (Valencia, California), per manufacturer's instructions. A segment of E. typhina tubB (previously tub2), including the first three introns, was amplified and sequenced from each sample as previously described (Craven et al. 2001) Sequences were aligned with Pileup (GCG 1998), with gap creation penalty = 0 and gap extension penalty $=1$. Alignments were adjusted by eye. Phylogenetic trees were inferred with PAUP* software (Swofford 1998), by parsimony with unweighted and unordered DNA character states, and indels treated as missing information.

\section{Results}

Each E. typhina isolate gave a single $t u b B$ haplotype, in keeping with the haploid nature of this species (Fig. 1). In total, $4 \mathrm{tubB}$ haplotypes were identified in the 38 isolates from $B$. pinnatum, 3 haplotypes in 26 isolates from $P$. nemoralis, and 2 haplotypes in 79 isolates from $P$. trivialis. The most genetic diverse group was that associated with D. glomerata, with a total of 24 haplotypes identified in 117 isolates.

Haplotypes in isolates from P. nemoralis and P. trivialis formed distinct clades, but despite the generic relationship of the hosts, these clades were not closely related. Surprisingly, populations associated with $D$. glomerata and $B$. pinnatum did not form monophyletic clades (Fig.2). Instead, those from $B$. pinnatum were paraphyletic to the rest of the E. typhina complex, and those from $D$. glomerata were paraphyletic to the P.trivialis-associated clade, as well as to sequences in isolates from the hosts Lolium perenne, Anthoxanthum oderatum, and Holcus lanatus (the latter are classified as E. clarkii). Some isolates from B. pinnatum had haplotypes that were very similar (each with a single nucleotide substitution difference) to haplotypes of E. sylvatica.

\section{Discussion}

The lack of any shared haplotypes in our study strongly indicates that E. typhina populations associated with different host species rarely or never mate productively. Mating of the Epichloë species are mediated by species of Botanophila, anthymiid flies that vector spermatia between stromata, and which depend on the resulting ascomal development for larval nutrition (Bultman et al. 1995). However, analysis of conidia from frass indicates that the flies are not entirely specific for stromata on individual host species (Leuchtmann \& Bultman 2001). Therefore, another genetic isolation mechanism may be at work. A possibility is that hybrids from matings of E. typhina strains on different hosts are rarely capable of survival in one or the other parental host. This hypothesis was largely supported by a study of host compatibility of progeny obtained when $E$. typhina from $D$. glomerata was mated with E. typhina from L. perenne (Chung et al. 1997). However, host compatibility appeared to be a complex, quantitative trait. Furthermore, such matings gave rise to some recombinant ascospores that infected L. perenne (Chung \& Schardl 1997), and even a hybrid that was capable of producing stromata on D. glomerata (Chung et al. 1997). Thus, past studies suggest that host species barriers are not absolute, although such bariers are apparently sufficient to prevent substantial genetic exchange between host-specialised populations. The relative diversity and paraphyletic relationships of populations on B. pinnatum and D. glomerata suggest that $E$. typhina parasitism of those hosts may be relatively ancient, and that populations on other hosts arose by jumps or shifts. Some of these jumps were between hosts in different tribes, such as may have occurred between Brachypodium spp. (Brachypodieae) and Poa spp. (Poeae). In other cases it is unclear if host jumps occurred or, instead, if E. typhina populations diverged along with the speciation of the hosts. So, for example, it is possible that the closely related isolates of E. sylvatica on B. sylvaticum and E. typhina on B. pinnatum may represent cocladogenesis with the host grasses. The lack of detectable genetic exchange between populations on different hosts suggests cryptic speciation driven by host specialisation. However, the instances of paraphyly pose a problem for phylogenetically based recognition of such species (Taylor et al. 2000). Such paraphyletic relationships may be expected if new niches (in this case, new hosts) are colonized rarely, and by few individuals from larger ancestral populations (founder effect), and if hybrids between individuals specialised on different hosts are strongly disfavoured. The large number of host species with epichloë endophytes suggests that colonisation of new hosts is common on evolutionary time scales. However, the majority of such endophytes are strictly asexual, and are maintained by vertical transmission in host seeds. Perhaps the majority of host jumps result in asymptomatic symbioses, and relatively few give symbioses in which the endophyte can express the sexual state (choke). Indeed, many asexual endophytes appear to be derived either from E. typhina or from diploid or polyploid hybrids possessing an E. typhina-derived genome (Gentile et al. 2005; Moon et al. 2004). Ironically, most of the hosts that support stroma formation by E. typhina do not support vertical transmission. In such cases, stromata are necessary to provide the inoculum (ascospores) to spread the fungus in the host population. The only known exception to date is $P$. nemoralis, on which E. typhina is capable of both vertical and horizontal transmission (Schardl \& Leuchtmann 2005). Not surprisingly, E. typhina genotypes on $P$. nemoralis are closely related to non-hybrid asexual endophytes in several hosts (Gentile et al. 2005; Moon et al. 2004). In contrast, jumps to new hosts of other E. typhina genotypes will probably be dead ends unless the sexual state is expressed on the new hosts (or the endophyte hybridises with a resident, vertically transmissible endophyte). Thus, we speculate that a major selection for host specialisation is the requirement for stromal expression to maintain a viable, contagious population.

\section{ACKNOWLEDGEMENTS}

This study was supported by a Visiting Professorship stipend provided to CLS by the ETH Zürich, Switzerland.

\section{REFERENCES}

Bultman, T.L.; White, J.F., Jr.; Bowdish, T.I.; Welch, A.M.; Johnston, J. 1995. Mutualistic transfer of Epichloë spermatia by Phorbia flies. Mycologia 87: 182-189.

Chung, K.R.; Schardl, C.L. 1997. Sexual cycle and horizontal transmission of the grass symbiont, Epichloe typhina. Mycological Research 101: 295-301.

Chung, K.R.; Hollin, W.; Siegel, M.R.; Schardl, C.L. 1997. Genetics of host specificity in Epichloë typhina. Phytopathology 87: 599-605.

Craven, K.D.; Hsiau, P.T.W.; Leuchtmann, A.; Hollin, W.; Schardl, C.L. 2001. Multigene phylogeny of Epichloë species, fungal symbionts of grasses. Annals of the Missouri Botanical Garden 88: 14-34. 
GCG, 1998. Wisconsin Package, version 10.0. Genetics Computer Group (GCG), Madison, Wisconsin.

Gentile, A.; Rossi, M.S.; Cabral, D.; Craven, K.D.; Schardl, C.L. 2005. Origin, divergence, and phylogeny of epichloë endophytes of native Argentine grasses. Molecular Phylogenetics and Evolution 35: 196-208.

Leuchtmann, A. 1994. Isozyme relationships of Acremonium endophytes from twelve Festuca species. Mycological Research 98: 25-33.

Leuchtmann, A.; Bultman, T.L. 2001. Epichloë grass endophytes and their interaction with a symbiotic fly. pp. 97-101. In: Proceedings of the 4th International Neotyphodium/Grass Interactions Symposium. Eds. Paul, V. H.; Dapprich, P. D. Fachbereich Agrarwirtschaft, Soest, Germany.

Leuchtmann, A.; Clay, K. 1988. Atkinsonella hypoxylon and Balansia cyperi, epiphytic members of the Balansiae. Mycologia 80: 192-199.

Leuchtmann, A.; Schardl, C.L. 1998. Mating compatibility and phylogenetic relationships among two new species of Epichloë and other congeneric European species. Mycological
Research 102: 1169-1182.

Moon, C.D.; Craven, K.D.; Leuchtmann, A.; Clement, S.L.; Schardl, C.L. 2004. Prevalence of interspecific hybrids amongst asexual fungal endophytes of grasses. Molecular Ecology 13: 1455-1467.

Schardl, C.L.; Leuchtmann, A. 2005. The epichloë endophytes of grasses and the symbiotic continuum. pp. 475-503 In: The Fungal Community: its Organization and Role in the Ecosystem. Eds. Dighton, J.; White, J. F.; Oudemans, P. CRC Press, Boca Raton, Florida.

Swofford, D.L. 1998. PAUP*. Phylogenetic Analysis Using Parsimony (*and Other Methods). Sinauer Associates, Sunderland, Massachusetts.

Taylor, J.W.; Jacobson, D.J.; Kroken, S.; Kasuga, T.; Geiser, D.M.; Hibbett, D.S.; Fisher, M.C. 2000. Phylogenetic species recognition and species concepts in fungi. Fungal Genetics and Biology 31: 21-32.

White, J.F., Jr. 1993. Endophyte-host associations in grasses. XIX. A systematic study of some sympatric species of Epichloë in England. Mycologia 85: 444-455. 